\title{
Circadian clocks and breast cancer
}

\author{
Victoria Blakeman ${ }^{\dagger}$, Jack L. Williams ${ }^{\dagger}$, Qing-Jun Meng ${ }^{*}$ and Charles H. Streuli ${ }^{*}$
}

\begin{abstract}
Circadian clocks respond to environmental time cues to coordinate 24-hour oscillations in almost every tissue of the body. In the breast, circadian clocks regulate the rhythmic expression of numerous genes. Disrupted expression of circadian genes can alter breast biology and may promote cancer. Here we overview circadian mechanisms, and the connection between the molecular clock and breast biology. We describe how disruption of circadian genes contributes to cancer via multiple mechanisms, and link this to increased tumour risk in women who work irregular shift patterns. Understanding the influence of circadian rhythms on breast cancer could lead to more efficacious therapies, reformed public health policy and improved patient outcome.
\end{abstract}

\section{Background}

The global impact of breast cancer is large and growing. With 1.67 million cases reported in 2012, it is the second most commonly diagnosed cancer worldwide $[1,2]$. The incidence of the disease is much higher in the developed world, with four times as many cases in Western Europe as in Middle Africa and Eastern Asia. This suggests that aspects of a modern western lifestyle may influence the onset and progression of breast cancer. One possibility is a disruption to our internal body clocks, known as circadian clocks [3].

Intrinsic circadian clocks are driven by environmental time cues such as the natural day/night cycle. Our bodies translate timing cues into molecular oscillations within individual cells, which then drive 24-hour rhythms in cellular processes in almost every tissue in the body [4-6]. These cell-autonomous molecular oscillators make up the body's internal timing system, and are synchronized by the master pacemaker, the suprachiasmatic nucleus (SCN) [7].

\footnotetext{
* Correspondence: qing-jun.meng@manchester.ac.uk; cstreuli@manchester.ac.uk ${ }^{\dagger}$ Equal contributors

Faculty of Biology, Medicine and Health, and Wellcome Trust Centre for Cell-Matrix Research, University of Manchester, Oxford Road, Manchester M13 9PT, UK
}

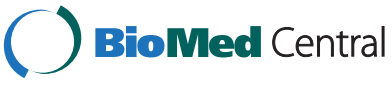

(c) 2016 The Author(s). Open Access This article is distributed under the terms of the Creative Commons Attribution 4.0 International License (http://creativecommons.org/licenses/by/4.0/), which permits unrestricted use, distribution, and reproduction in any medium, provided you give appropriate credit to the original author(s) and the source, provide a link to the Creative Commons license, and indicate if changes were made. The Creative Commons Public Domain Dedication waiver (http://creativecommons.org/publicdomain/zero/1.0/) applies to the data made available in this article, unless otherwise stated.
However, circadian clocks can become perturbed through irregular shift work, through repeated bouts of jet lag and during ageing. Weakened or damaged circadian clocks alter the susceptibility to certain diseases and directly drive others.

One of the processes regulated by the circadian clock is the cell cycle. Disruption of circadian rhythms can therefore be associated with abnormal cell divisions that occur in cancer [8]. Indeed, there are links between altered circadian clocks and tumorigenesis in metastatic colorectal cancer, osteosarcoma, pancreatic adenocarcinoma and, most notably, breast cancer [8].

The influence of altered circadian rhythm on breast cancer was first noted in the 1960s [9]. Since then, it has become clear that circadian disruption interrupts the complex multi-step molecular mechanisms underpinning breast cancer [1]. The indication that tumorigenesis is linked to circadian rhythms suggests that manipulating those rhythms might be a remedial approach for treating cancer. This could, for example, lead to more efficacious therapies, novel adjuvant strategies and, ultimately, improved breast cancer outcome [10].

In this review, we discuss the current understanding of links between circadian disruption and breast cancer risk.

\section{The SCN as a central pacemaker}

Located in the anterior hypothalamus, the SCN is the central pacemaker that coordinates circadian rhythms with the solar day [11]. The bilateral SCN receives innervation directly from the retina via the retinohypothalamic tract. The majority of its $\sim 20,000$ densely packed neurons are 'pacemaker cells', with each neuron containing its own oscillatory machinery capable of producing a prolonged and robust circadian rhythm even in ex-vivo culture [12].

Light is a dominant synchronizing time-giver. However as well as light/dark cycles, the SCN is also responsive to changes in rest/activity cycles [5]. Non-photic stimuli, such as neuroendocrine signals and feeding behaviour, can also influence SCN pacemaking [5]. The $\mathrm{SCN}$ uses several neural and endocrine outputs to synchronize clocks of many peripheral organs [6]. One 
of these is the hormone melatonin [13], which is released rhythmically at night and relays information to peripheral organs [14].

The SCN is not actually required for peripheral organs to generate their own rhythms. Rather, it acts more like the conductor of an orchestra, guiding each organ to oscillate in the ideal phase for that specific tissue [15].

\section{Circadian clock genes and the circadian cycle}

The basic genetic regulation of the circadian clock is highly conserved across the animal kingdom. Most mammalian clock genes were first identified via mutagenic studies in fruit flies [16]. The core molecular clock generates oscillations in protein levels via a series of auto-regulatory transcriptional/translational feedback loops [17]. Several clock genes encode transcription factors, with the molecular clock driving rhythmic expression of downstream clock-controlled genes [18].

The major components involved in this cellular clock network include the transcriptional activators Circadian Locomotor Output Cycles Kaput (CLOCK) and Brain and muscle Arnt-like protein-1 (BMAL1). CLOCK has a paralogue, Neuronal PAS domain protein 2 (NPAS2), which compensates for loss of CLOCK in the SCN and peripheral oscillators $[19,20]$. The other main components are Period (PER1 and PER2) and Cryptochrome (CRY1 and CRY2), which form the negative arm of a feedback loop [17]. Additional regulatory systems such as nuclear hormone receptors and epigenetic mechanisms have also been identified [21, 22].

Circadian gene expression is the result of a series of transcription and translation events. This leads to the expression of a different set of proteins that switches them off at the end the day. The process then begins again with timely removal of the repressors (PERs and CRYs).

At the beginning of the circadian day, Bmal1 expression is driven by $\mathrm{ROR} \alpha$, allowing the formation of CLOCK/BMAL1 heterodimers (Fig. 1). This transcription complex binds to CACGTG E-box sequences in the promoters of the Per and Cry genes, increasing their expression. The CLOCK/BMAL1 complex also increases expression of Rev-erb $\alpha$, which suppresses Bmal1 transcription [23]. The CLOCK/BMAL1-mediated increase in Per and Cry expression allows for the accumulation of PER in the cytosol, where it is phosphorylated by casein

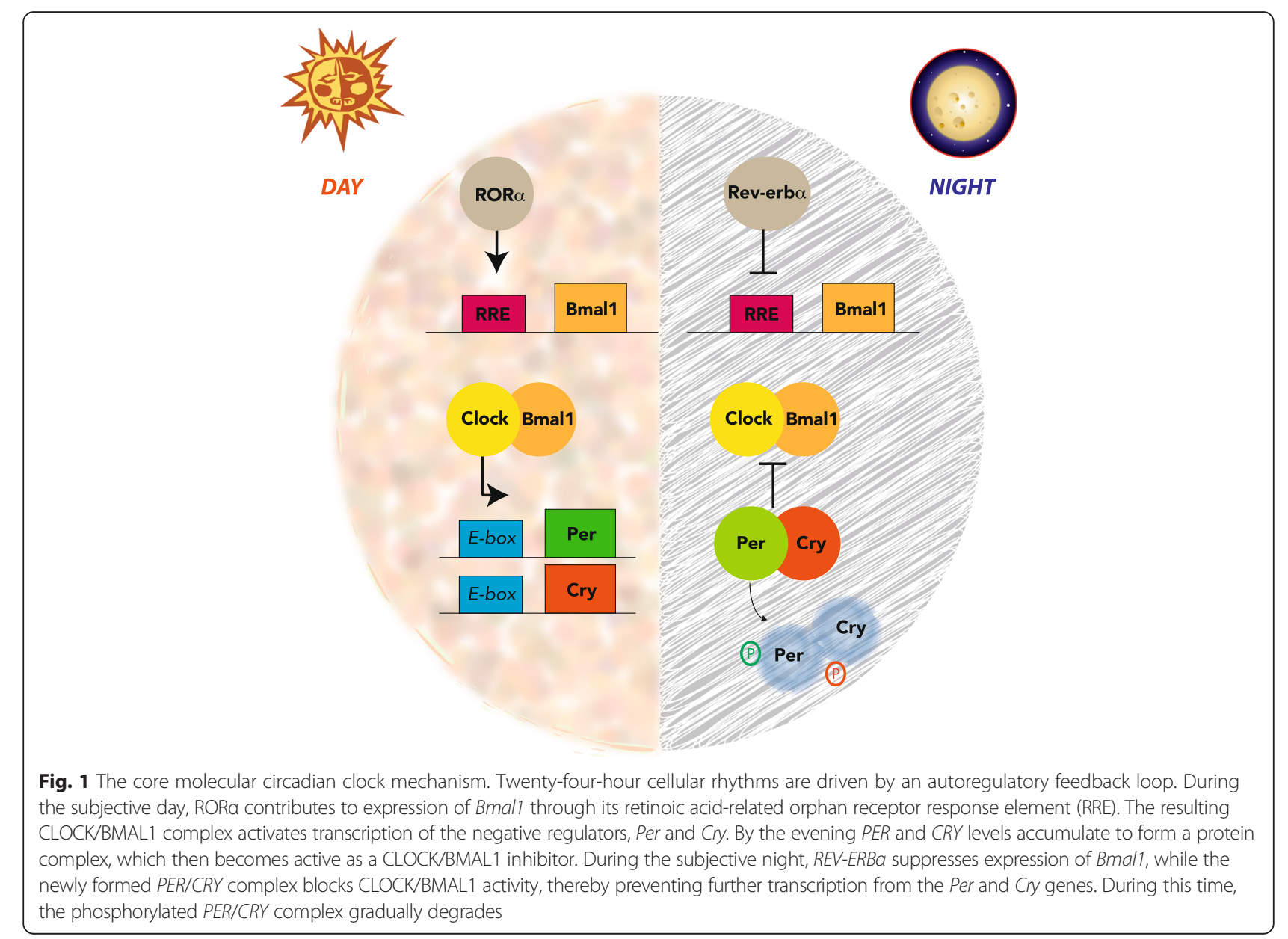


kinase $1 \varepsilon$ and $1 \delta$. Phosphorylated PER becomes ubiquitinated and is readily degraded, but CRY accumulation allows the formation of a stable PER/CRY/CK1 complex [7]. This complex inhibits the transcriptional capacity of CLOCK/BMAL1, preventing further expression of Per and Cry, and also Rev-erba. Eventually phosphorylated PER and CRY are lost, de-repressing Bmal1 transcription and allowing for higher levels of BMAL1 that start the next circadian day [18].

Collectively these cycles ensure that the circadian clock oscillates with a period of 24 hours, driven by the appropriate environmental time cues (Fig. 1).

\section{Peripheral circadian clocks}

Peripheral tissues have their own intrinsic self-sustained circadian oscillations, but are dependent on the central clock and tissue-specific factors for synchronization. Local cellular oscillators regulate specific circadian programmes of gene expression that vary according to tissue function, with typically $5-10 \%$ of all genes being transcribed rhythmically [24].

Clock target genes can be controlled directly by the CLOCK/BMAL1 complex, or indirectly via the circadian expression of transcription factors. In this way, the circadian clock confers a rhythmic aspect to a wide range of tissues. In the breast, for example, the expression of nearly 600 genes is controlled in a circadian manner.

The circadian regulation of gene expression impacts upon many cellular processes and complex behaviours. This is exemplified by SIRT1 and TIMELESS, proteins that independently intersect the clock and the cell cycle machines. For example, SIRT1 reduces proliferation by deacetylating $\beta$-catenin [25] and binding p53 [26], whereas TIMELESS advances the circadian clock in response to DNA damage $[27,28]$.

The circadian system is therefore well integrated with other physiologies. If the clock becomes disrupted, however, this can lead to disease [5].

\section{Clock genes in breast biology}

There is growing evidence that circadian clock gene expression plays a role in breast biology. In mice, mutations of specific circadian genes disrupt behavioural and molecular rhythms. Such mutations also reveal the involvement of cellular clocks in the initiation of carcinogenesis.

Breast tissue contains a network of branched epithelial ducts surrounded by a basement membrane, outside which is a fibroblast-rich and adipose-rich stromal extracellular matrix [29]. Clocks have been discovered within breast epithelium using real-time bioluminescent imaging of mammary explants from PER2::Luciferase [30] mice. There are daily rhythmic variations in the expression of core clock proteins BMAL1 and PER2. Moreover, during the development of the gland from virgin through to full lactation, tissue isolated at the same time of day reveals that Bmal1 and Per1 mRNA levels increase in late pregnancy and lactation, while Per2 falls [31, 32].

The levels of clock gene expression are also controlled by the breast tissue microenvironment. For example, we have found that a stiff extracellular matrix such as is found in ageing and cancer leads to suppression of core clock rhythms. Intriguingly, local stiffness of the breast stroma and extracellular matrix adjacent to breast epithelium has a major role in determining cancer outcome $[33,34]$. The mechanisms linking the cell exterior to clock control are not yet known, but could involve the cytoskeleton and/or nuclear envelope proteins, one of which regulates transcription of the Bmal1 gene [35].

The molecular clock is also crucial for regulating the survival of stem cells. Indeed the self-renewal capacity of mammary progenitor stem cells becomes compromised in mice that have a defective CLOCK/BMAL1 complex, revealing a circadian influence on breast function [36]. Studies in other tissues have demonstrated similar links between the circadian clock and stem cells. For example, undifferentiated neural stem cell cultures from BMAL1 ${ }^{-/-}$ mice have an increased propensity to differentiate into glia over neurons [37]. In addition, circadian oscillations in the release of hematopoietic stem cells (HSCs), through chemokine Cxcl12 and GSK3 $\beta$, could result in a coordinated release of cells and thereby repopulation of the bone marrow stem cell niche [38].

Clock genes are expressed in normal breast. However, their levels are variable and controlled both by the cellular microenvironment and by the developmental stage of the tissue. Disruption of clock gene expression can increase breast cancer risk (Table 1).

\section{The role of circadian genes in breast cancer Altered cell cycle and apoptosis}

Per mutant mice have revealed a role for circadian gating of cells passing through the cell cycle. Indeed, an altered Per gene contributes to malignancy. In humans, there is reduced expression of Per genes in both sporadic and familial breast cancer cells compared with normal breast, perhaps occurring via methylation in regions of the Per promoter [1]. In mice, Per2 loss-of-function mutations exhibit higher tumour incidence and show greater susceptibility to radiation-induced malignant lymphoma compared with wild type [39].

Both PER1 and PER2 also promote apoptosis. Indeed they may suppress breast cancer in vivo by inducing apoptosis [4] (Fig. 2). However, if there is decreased expression of both Per1 and Per 2 in breast tumours the action of PER as a tumour suppressor becomes reduced. Moreover they can indirectly suppress $c-M y c$ transcription by inhibiting E-box-mediated transactivation by BMAL1/Npas2 [32]. 
Table 1 Genetic associations between circadian clocks and breast cancer

\begin{tabular}{|c|c|c|}
\hline Mutation/SNP & Possible mechanism & Phenotype \\
\hline CLOCK & & $\begin{array}{l}\text { Self-renewal capacity of mammary progenitor cells } \\
\text { becomes compromised (our unpublished data) }\end{array}$ \\
\hline $\begin{array}{l}\text { Hypermethylation of Clock } \\
\text { promoter }\end{array}$ & Mediates CCL5 expression & Reduced breast cancer risk [36] \\
\hline NPAS2 Ala394Thr SNP & Altered NPAS2 protein structure & Increased breast cancer risk [55] \\
\hline Perl deficient & Alters expression of checkpoint proteins ATM and Chk2 & Increased proliferation [41] \\
\hline Per1 overexpression & $\begin{array}{l}\text { Impairs p53 leading to decreased apoptosis, deregulation } \\
\text { of c-myc/CyclinD1/Gadd } 45\end{array}$ & $\begin{array}{l}\text { Reduces proliferation in colon, lung and breast cancer } \\
\text { cell lines [41] }\end{array}$ \\
\hline Per2 deficient & $\begin{array}{l}\text { Increases OCT1 binding to EMT genes Slug, Snail and } \\
\text { Twist1 }\end{array}$ & $\begin{array}{l}\text { Higher tumour incidence, increased susceptibility to } \\
\text { radiation-induced malignant lymphoma [39] }\end{array}$ \\
\hline Per2 overexpression & Cell cycle arrest, growth inhibition, apoptosis induction & Suppresses breast cancer in vivo $[40,41]$ \\
\hline Per3 deficient & & Higher probability of cancer recurrence $[7,62]$ \\
\hline Cry deficient & $\begin{array}{l}\text { Disrupted cell cycle regulation via de-regulation of Wee-1 } \\
\text { and CyclinD1 }\end{array}$ & \\
\hline BMAL1/Era/Per2 KO & Prevents mammary acinar formation & Facilitates invasion and metastasis [56] \\
\hline BMAL1 overexpression & Binds to $p 53$ promoter & Tumour suppression [61] \\
\hline
\end{tabular}

Circadian mutations covered in this review and their links to cancer. Both epidemiological and experimental data are included, along with possible mechanisms and resultant phenotypes, where known

KO knockout, SNP single nucleotide polymorphism

Loss of Per2 leads to decreased apoptosis, and therefore accumulation of damaged cells, by impairing p53 [40]. Consistent with this, Per 2 overexpression in colon cancer results in cell cycle arrest, growth inhibition and apoptosis [41]. Additionally, Per2 mutants exhibit deregulation of $c$ Myc, and its target cell-cycle genes CyclinD1 and Gadd45 $[39,42,43]$. Similarly, inhibition of PER1 alters expression of key cell cycle regulators, by interacting with checkpoint proteins ATM and Chk2, and Per1 overexpression can reduce proliferation in colon, lung and breast cancer cell lines [41]. Intriguingly in U2OS cells, MYC can regulate the circadian system by binding to E-box elements. High levels of MYC expression dampen the circadian clock and promote proliferation [43].

CRY proteins also impact tumorigenesis via the cell cycle. The cell cycle suppressor WEE-1 is expressed in phase with PER, during times of day when entry to the $M$ phase is suppressed [44]. However, mice deficient in the Cry genes have de-regulated Wee-1 and CyclinD1, and therefore exhibit disrupted cell cycle regulation. So far this has been shown in liver cells, but has yet to be explored in breast tumours.

Disruption of PER and CRY therefore causes downregulation of growth control genes, implying a mechanistic link between the circadian system and cell proliferation. Moreover, these findings indicate that the circadian clock is involved with both cell cycle control and apoptosis.

\section{Altered cell metabolism}

The circadian clock can also initiate and propagate cancer through its effects on cell metabolism (Fig. 2). Metabolic regulators SIRT1 and AMPK are cellular switches that alter cell behaviour according to metabolic state, and both have been linked to the circadian clock [45-47]. SIRT1 is a histone deacetylase that is active under high levels of cellular $\mathrm{NAD}^{+}$, and inactive under high levels of NADH [48]. SIRT1 deacetylates p53, inhibiting its activity and reducing apoptosis, which could have implications in cancer [49]. The cellular ratio of $\mathrm{NAD}^{+}$and the deacetylase activity of SIRT1 are both under circadian control [50]. They can feed back into the core clock machinery and thereby regulate the circadian clock [51].

The cellular energy state is reflected in the ratio of AMP + ADP:ATP. When ATP levels are low, AMPK is phosphorylated by an upstream kinase [52]. In its active, phosphorylated state, AMPK regulates many processes, including glucose uptake, mitochondrial biogenesis, cell proliferation and the circadian clock [53]. The cellular clock regulates AMPK activity which in turn induces degradation of the components of the negative arm of the core clock loop, directly phosphorylating Cry1 and inducing CK1-mediated degradation of Per2 [47, 54].

Clock disruption could directly impact SIRT1 and AMPK signalling pathways, both of which are crucial in controlling cell proliferation, apoptosis and tumour suppressor pathways. Furthermore, global disruption of circadian behaviour by shift work, jet lag and ageing can affect the feeding schedule of an individual. This could lead to energy imbalances across the circadian cycle and further drive detrimental activity through these signalling pathways.

\section{Single nucleotide polymorphisms}

Breast cancer risk is also associated with single nucleotide polymorphisms (SNPs) in Npas2 and Cry2, as well as Clock [36]. Hypermethylation of the Clock promoter 


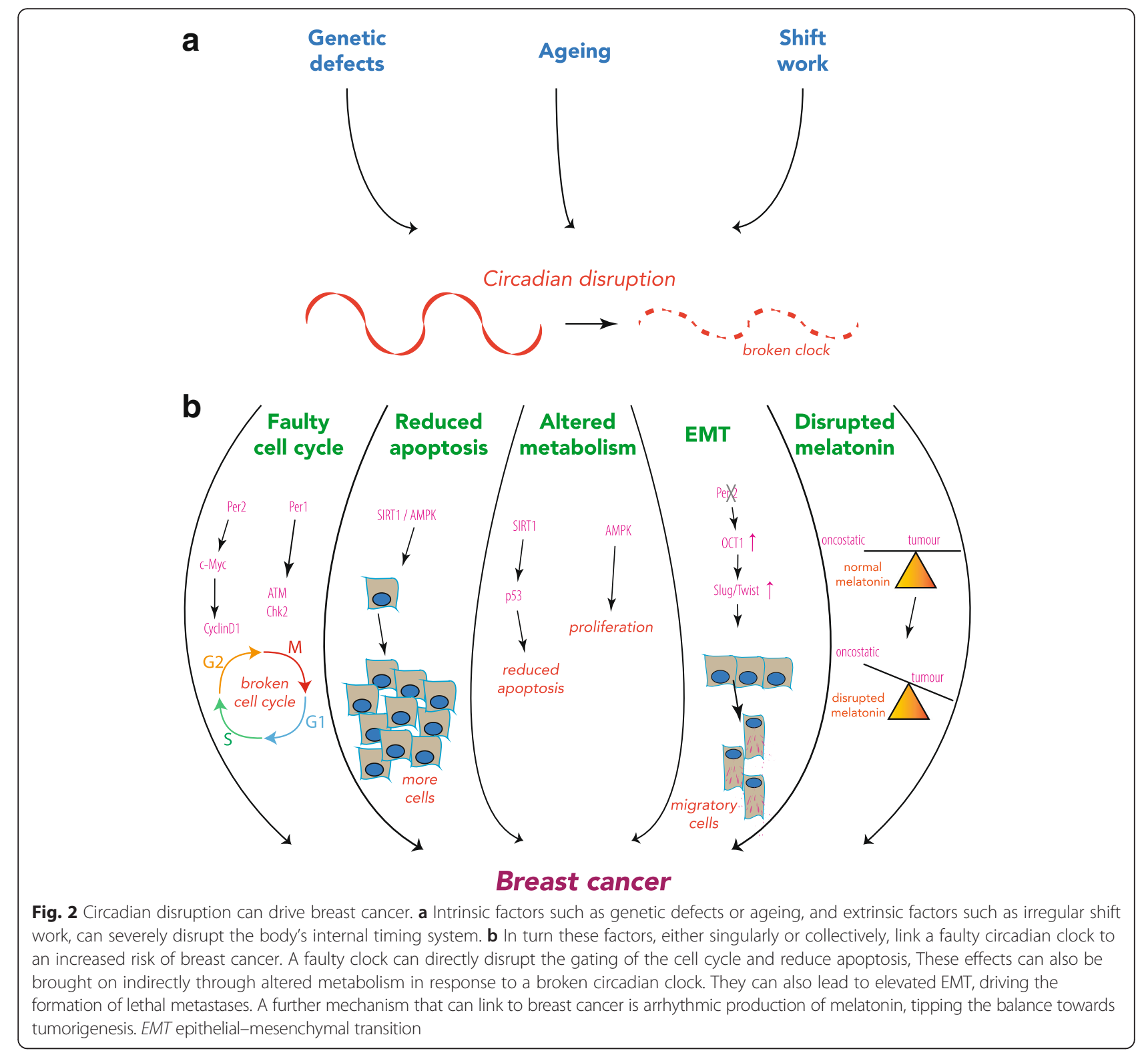

reduces breast cancer risk, with lower levels of CLOCK in healthy controls. SNPs in Clock directly alter the expression of genes linked to cell cycle progression, such as CCL5. Similarly, Npas 2 polymorphisms are associated with increased breast cancer risk. The Ala394Thr SNP alters the NPAS2 protein structure, which interferes with NPAS2/BMAL1 heterodimerization. Women with this SNP are at significantly higher risk of developing the disease ([55], Table 1).

\section{Link to oestrogen receptor}

Per gene expression correlates with other genes implicated in breast cancer, for example that encoding the oestrogen receptor (ER). Oestrogen receptor interacts with PER2 and BMAL1 and is integral to the formation of mammary acini, which are the core cellular structures within normal breast [56]. Mammary epithelial cells are highly polarized, and proteins are differentially expressed across the cells [57]. The polarity of the cells and acinar stability are vital for directional milk secretion into alveolar lumens [29]. However, knocking down PER2, BMAL1, or ER prevents acinar formation, perhaps via a feedback loop between oestrogen and the clock [56].

PER1 also influences $E R$ transcriptional regulation, while PER2 interacts with ER to suppress oestrogen-mediated transcription of ER target genes [58]. Because Per is also induced by oestrogen, there is a feedback loop coupling the circadian clock and the oestrogen pathway. Absent $E R$ expression is associated with an aggressive tumour phenotype, and dysregulation of ER-transcriptional activity can 
equally lead to breast cancer [59]. PER may also act with the breast cancer protein, BRCA1, to regulate $E R$ transcription [60].

\section{Metastasis and epithelial-mesenchymal transition}

Low-grade and non-metastasizing tumours maintain functional circadian clocks. In more aggressive carcinomas, however, the coordinated circadian oscillation of clock genes becomes compromised [7]. For example, Bmal1 expression in the pancreas is significantly reduced in tumours, correlating with cancer severity [61]. In the breast, PER and $\mathrm{CRY}$ are associated with better prognosis in $\mathrm{ER}^{+} / \mathrm{HER}^{-}$ tumours, yet CLOCK and NPAS2 are linked to better prognosis in the more aggressive $\mathrm{ER}^{-} / \mathrm{HER} 2^{-}$tumours. Furthermore, high expression of Clock, Per and Cry is associated with longer metastasis-free survival [7]. In contrast, loss of expression of the Per3 gene equates with tumours of ER negativity, high histological grade and higher probability of recurrence [62]. Indeed, loss of PER3 and CRY2 co-expression is associated with a higher risk of breast cancer metastasis [7].

While altered levels of clock proteins may affect the tissue structure and contribute to cellular transformation, they also influence an epithelial-mesenchymal transition (EMT) and thereby facilitate invasion and metastasis (Fig. 2). For example, loss of PER2 could directly drive EMT through OCT1. Under normal conditions, PER2 recruits transcriptional co-repressors to OCT1binding promoters of EMT genes Twist1, Slug and Snail. However, PER2 becomes deregulated in hypoxic, tumourlike conditions, allowing EMT gene expression to be activated [42].

\section{Summary of the role of the clock in breast cancer}

Together these studies show that clock gene defects in mammary epithelium can lead to cell cycle disruption. This disruption causes improper cell division, increased susceptibility to breast cancer and leads to more aggressive tumours. Although there are central roles for circadian genes in normal breast biology, in the following we discuss how disrupting the normal light/dark cycle can also cause disease.

\section{Shift work, night-time light exposure and breast cancer}

Over the last 20 years, epidemiological evidence has corroborated a link between altered clocks and breast cancer. Women who work irregular shift patterns, such as nurses, have a higher frequency of hormone-related breast cancer [63-69], and the higher levels of cancer may be greater for women who started night shifts before a first pregnancy [70]. Elevated breast cancer risk also occurs in women exposed to high levels of ambient light at night [71]. Irregular shift work refers to working a mix of nights and days during a week. In these individuals, the circadian clock has too little time to entrain to the new shift pattern before it is changed again. Notably, however, working consistently at night causes far less disruption to the circadian rhythm.

Mice subjected to a simulated shift-work paradigm have a significantly increased risk of acquiring mammary tumours [72]. By inverting the light cycle every week, mice acquired tumours faster than age-matched littermates housed under normal light/dark cycles. Disruption of sleep patterns, and thereby the internal body clock, of shift workers therefore directly influences their physiology and the rate of cancer development. Moreover, working night shifts three or more times per month elevates the risk of breast cancer in humans. This risk increases with age and with more hours per week of night-shift work [63, 66, 67, 73].

Constant light exposure during the night disturbs circadian pacemaker activity in the mammalian $\mathrm{SCN}$. This ultimately disrupts circadian rhythms throughout the body. One mechanism may be through altered production of the hormone melatonin. Night-time light exposure reduces the rhythmic secretion of melatonin from the pineal gland [74]. This hormone has oncostatic activity in experimental animals with mammary tumours, and also in human breast cancer cells in culture [75] (Fig. 2). Melatonin promotes genomic stability, and may also have anti-invasive and anti-metastatic activity [76]. For example, in endocrine-responsive cancer, melatonin reduces the expression and activity of aromatase, which normally converts testosterone to oestrogen. The oncostatic action of melatonin on hormone-dependent mammary tumours is mainly based on its anti-estrogenic actions, both reducing biosynthesis of oestrogen from androgens and neutralizing the cellular effects of oestrogen. Rats exposed to small amounts of light at night, rather than no light, had disrupted melatonin profiles and fastergrowing mammary tumours that were tamoxifen resistant; this could be reversed by melatonin supplementation [77]. In humans, reduction of nocturnal melatonin levels may increase the effects of oestrogen and thereby contribute to breast cancer risk-and indeed, make tumours insensitive to steroid therapy. Epidemiological studies in nurses also reveal that shift work induces a significant increase in circulating oestradiol levels, which could further disrupt mammary oestrogen signalling and thereby promote cancer [78].

Another potential mechanism linking altered light exposure at night-time to breast cancer risk could be via the circadian regulation of micro-RNA (miRNA) expression. Several miRNAs known to be involved with breast cancer risk show fluctuations during the day. For example, the levels of miR-150-5p and miR-133a-3p are dramatically altered by circadian disruption [79]. Some 
of these miRNAs are interconnected with the expression of proteins known to have roles in breast cancer, such as NFkB and Stat3. The levels of some transcripts that encode proteins involved with cancer are also altered in circadian-disrupted mice [80].

Night-time light exposure can also happen as a result of loss of rhythmic behaviour, such as in SCN-lesioned animals. This both increases the risk of tumour formation and enhances progression of pre-existing tumours. For example, in SCN-ablated mice that are inoculated with implants of osteosarcoma or pancreatic adenocarcinoma, tumours grow two to three times faster than in sham-operated comparators [8]. In the breast, a robust circadian rhythm is associated with slow-growing tumours, while altered rhythms are linked to faster growing tumours $[81,82]$.

Together the evidence suggests that disrupting circadian clocks via night-time light exposure has an adverse role on tumour progression. This applies to those working irregular shift patterns, although it is still controversial whether there are links in flight attendant jet lag [83] or whether the widespread use of electric lighting at night might contribute to breast cancer [84].

The mechanisms by which altered light exposure contributes to cancer are not yet understood fully. However, the link between circadian disruption and breast cancer may have profound societal importance.

\section{Conclusion}

Tissue-based oscillators within the breast are coordinated by the $\mathrm{SCN}$, and are influenced by photic, endocrine, neural and metabolic cues. Analysis of the strong circadian component within the breast has highlighted its influence on the cell cycle, which impacts on tumour progression and carcinoma aggression.

In-vivo evidence has revealed the role of the circadian molecular clockwork in tumour suppression, highlighting that breast cancer should no longer be treated as a local disorder but rather can result from systemic defects in tissue control.

Mouse models have demonstrated the importance of core clock genes within the breast, whether that is in progenitor cell renewal, tumour incidence, or interaction with the oestrogen pathway. Collectively, this indicates that there is an oncostatic role of some clock genes.

Together with the epidemiological data linking an increased incidence of breast cancer in shift workers, this has serious implications for the demands of modern society. Circadian disruption imposes a major public health issue that has yet to receive the recognition it deserves. Nocturnal lifestyles perturb the normal patterns of circadian behaviour, resulting in detrimental effects on clock-controlled physiological and metabolic pathways. Increased public awareness of the circadian influences on breast cancer risk could enhance lifestyle choices, as well as improve the alignment of physiological systems with the daily body clock.

There is still a fair way to go in characterizing the molecular basis of how the circadian system influences breast cancer, and which of the different genetic types of breast cancer are most influenced by altered clocks. Further delineation of the mechanisms linking clock genes to cancer is needed in order to fully understand how an altered circadian system and how rotating shift work influence the disease. This knowledge will help to improve future therapeutic interventions.

\section{Abbreviations}

BMAL1, Brain and muscle Arnt-like protein-1; CLOCK, Circadian Locomotor Output Cycles Kaput; CRY, Cryptochrome; EMT, epithelial-mesenchymal transition; ER, oestrogen receptor; miRNA, micro-RNA; NPAS2, Neuronal PAS domain protein 2; PER, Period; SCN, suprachiasmatic nucleus; SNP, single nucleotide polymorphism

\section{Acknowledgements}

Keith Brennan kindly read the manuscript.

\section{Funding}

A Career Development Award (G0900414) and a Centenary Early Career Award to QJM from the Medical Research Council, UK, supported this work. Core funding for the Wellcome Trust Centre for Cell-Matrix Research (088785/Z/09/Z) from the Welcome Trust, UK, also supported the work. A BBSRC DTP PhD studentship supported JW.

\section{Authors' contributions}

All authors contributed to writing this manuscript. All authors read and approved the final manuscript.

\section{Competing interests}

The authors declare that they have no competing interest.

\section{Consent for publication}

The funders had no role in the decision to publish or preparation of the manuscript.

Published online: 02 September 2016

References

1. Chen S-T, et al. Deregulated expression of the PER1, PER2 and PER3 genes in breast cancers. Carcinogenesis. 2005;26:1241-6.

2. Ferlay J, et al. Cancer incidence and mortality worldwide: sources, methods and major patterns in GLOBOCAN 2012. Int J Cancer J Int Cancer. 2015;136: E359-86.

3. Stevens RG. Circadian disruption and breast cancer: from melatonin to clock genes. Epidemiol Camb Mass. 2005;16:254-8.

4. Gery S, Koeffler HP. Circadian rhythms and cancer. Cell Cycle Georget Tex. 2010;9:1097-103.

5. Hastings $M H$, Reddy AB, Maywood ES. A clockwork web: circadian timing in brain and periphery, in health and disease. Nat Rev Neurosci. 2003;4:649-61.

6. Dibner $C$, Schibler $U$, Albrecht $U$. The mammalian circadian timing system: organization and coordination of central and peripheral clocks. Annu Rev Physiol. 2010;72:517-49.

7. Cadenas C, et al. Loss of circadian clock gene expression is associated with tumor progression in breast cancer. Cell Cycle Georget Tex. 2014;13:3282-91.

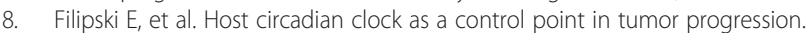
J Natl Cancer Inst. 2002;94:690-7.

9. Hamilton T. Influence of environmental light and melatonin upon mammary tumour induction. Br J Surg. 1969;56:764-6.

10. Reppert SM, Weaver DR. Molecular analysis of mammalian circadian rhythms. Annu Rev Physiol. 2001;63:647-76. 
11. Abrahamson EE, Moore RY. Suprachiasmatic nucleus in the mouse: retinal innervation, intrinsic organization and efferent projections. Brain Res. 2001; 916:172-91.

12. Welsh DK, Logothetis DE, Meister M, Reppert SM. Individual neurons dissociated from rat suprachiasmatic nucleus express independently phased circadian firing rhythms. Neuron. 1995;14:697-706.

13. Reiter RJ, Tan DX, Korkmaz A, Rosales-Corral SA. Melatonin and stable circadian rhythms optimize maternal, placental and fetal physiology. Hum Reprod Update. 2014;20:293-307.

14. Reiter RJ, Gultekin F, Manchester LC, Tan D-X. Light pollution, melatonin suppression and cancer growth. J Pineal Res. 2006;40:357-8.

15. Pando MP, Morse D, Cermakian N, Sassone-Corsi P. Phenotypic rescue of a peripheral clock genetic defect via SCN hierarchical dominance. Cell. 2002; 110:107-17.

16. Pittendrigh CS. Circadian systems. I. The driving oscillation and its assay in Drosophila pseudoobscura. Proc Natl Acad Sci U S A. 1967;58:1762-7.

17. Reppert SM, Weaver DR. Coordination of circadian timing in mammals. Nature. 2002;418:935-41.

18. Fu L, Lee CC. The circadian clock: pacemaker and tumour suppressor. Nat Rev Cancer. 2003;3:350-61.

19. Landgraf $D$, Wang $L L$, Diemer $T$, Welsh DK. NPAS2 compensates for loss of CLOCK in peripheral circadian oscillators. PLoS Genet. 2016;12:e1005882.

20. DeBruyne JP, Weaver DR, Reppert SM. CLOCK and NPAS2 have overlapping roles in the suprachiasmatic circadian clock. Nat Neurosci. 2007;10:543-5.

21. Gachon F, Olela FF, Schaad O, Descombes P, Schibler U. The circadian PARdomain basic leucine zipper transcription factors DBP, TEF, and HLF modulate basal and inducible xenobiotic detoxification. Cell Metab. 2006;4: 25-36.

22. Sahar S, Sassone-Corsi P. The epigenetic language of circadian clocks. Handb Exp Pharmacol. 2013;(217):29-44.

23. Preitner $\mathrm{N}$, et al. The orphan nuclear receptor REV-ERBalpha controls circadian transcription within the positive limb of the mammalian circadian oscillator. Cell. 2002;110:251-60

24. Reddy AB, Wong GKY, O'Neill J, Maywood ES, Hastings MH. Circadian clocks: neural and peripheral pacemakers that impact upon the cell division cycle. Mutat Res. 2005;574:76-91.

25. Firestein $\mathrm{R}$, et al. The SIRT1 deacetylase suppresses intestinal tumorigenesis and colon cancer growth. PLoS One. 2008;3:e2020.

26. Yi YW, et al. Targeting mutant p53 by a SIRT1 activator YK-3-237 inhibits the proliferation of triple-negative breast cancer cells. Oncotarget. 2013:4:984-94.

27. Unsal-Kaçmaz K, Mullen TE, Kaufmann WK, Sancar A. Coupling of human circadian and cell cycles by the timeless protein. Mol Cell Biol. 2005;25: 3109-16.

28. Engelen $\mathrm{E}$, et al. Mammalian TIMELESS is involved in period determination and DNA damage-dependent phase advancing of the circadian clock. PLoS One. 2013;8:e56623.

29. Muschler J, Streuli CH. Cell-matrix interactions in mammary gland development and breast cancer. Cold Spring Harb Perspect Biol. 2010;2: a003202.

30. Yoo S-H, et al. PERIOD2::LUCIFERASE real-time reporting of circadian dynamics reveals persistent circadian oscillations in mouse peripheral tissues. Proc Natl Acad Sci U S A. 2004;101:5339-46.

31. Metz RP, Qu X, Laffin B, Earnest D, Porter WW. Circadian clock and cell cycle gene expression in mouse mammary epithelial cells and in the developing mouse mammary gland. Dev Dyn Off Publ Am Assoc Anat. 2006;235:263-71.

32. Casey TM, et al. Tissue-specific changes in molecular clocks during the transition from pregnancy to lactation in mice. Biol Reprod. 2014;90:127.

33. McConnell JC, et al. Increased peri-ductal collagen micro-organization may contribute to raised mammographic density. Breast Cancer Res. 2016;18:5.

34. Sherratt MJ, McConnell J, Streuli CH. Raised mammographic density: causative mechanisms and biological consequences. Breast Cancer Res. 2016;18(1):45

35. Lin S-T, et al. Nuclear envelope protein MAN1 regulates clock through BMAL1. eLife. 2014:3:e02981.

36. Hoffman $A E$, et al. CLOCK in breast tumorigenesis: genetic, epigenetic, and transcriptional profiling analyses. Cancer Res. 2010;70:1459-68.

37. Malik A, Kondratov RV, Jamasbi RJ, Geusz ME. Circadian clock genes are essential for normal adult neurogenesis, differentiation, and fate determination. PLoS One. 2015;10:e0139655.

38. Méndez-Ferrer S, Chow A, Merad M, Frenette PS. Circadian rhythms influence hematopoietic stem cells. Curr Opin Hematol. 2009;16:235-42.
39. Fu L, Pelicano H, Liu J, Huang P, Lee C. The circadian gene Period2 plays an important role in tumor suppression and DNA damage response in vivo. Cell. 2002;111:41-50

40. Hua $\mathrm{H}$, et al. Circadian gene mPer2 overexpression induces cancer cell apoptosis. Cancer Sci. 2006;97:589-96.

41. Gery $\mathrm{S}$, et al. The circadian gene per1 plays an important role in cell growth and DNA damage control in human cancer cells. Mol Cell. 2006;22:375-82.

42. Kelleher FC, Rao A, Maguire A. Circadian molecular clocks and cancer. Cancer Lett. 2014;342:9-18.

43. Shostak A, et al. MYC/MIZ1-dependent gene repression inversely coordinates the circadian clock with cell cycle and proliferation. Nat Commun. 2016;7:11807.

44. Matsuo T, et al. Control mechanism of the circadian clock for timing of cell division in vivo. Science. 2003;302:255-9.

45. Asher G, et al. SIRT1 regulates circadian clock gene expression through PER2 deacetylation. Cell. 2008;134:317-28.

46. Xiao B, et al. Structure of mammalian AMPK and its regulation by ADP. Nature. 2011;472:230-3.

47. Lamia KA, et al. AMPK regulates the circadian clock by cryptochrome phosphorylation and degradation. Science. 2009;326:437-40.

48. Imai S, Armstrong CM, Kaeberlein M, Guarente L. Transcriptional silencing and longevity protein Sir2 is an NAD-dependent histone deacetylase. Nature. 2000;403:795-800.

49. Vaziri H, et al. hSIR2(SIRT1) functions as an NAD-dependent p53 deacetylase. Cell. 2001;107:149-59.

50. Nakahata Y, Sahar S, Astarita G, Kaluzova M, Sassone-Corsi P. Circadian control of the NAD+ salvage pathway by CLOCK-SIRT1. Science. 2009;324: $654-7$.

51. Nakahata Y, et al. The NAD + -dependent deacetylase SIRT1 modulates CLOCK-mediated chromatin remodeling and circadian control. Cell. 2008; 134:329-40

52. Hardie DG. Minireview: the AMP-activated protein kinase cascade: the key sensor of cellular energy status. Endocrinology. 2003;144:5179-83.

53. Hardie DG. AMP-activated protein kinase: an energy sensor that regulates all aspects of cell function. Genes Dev. 2011;25:1895-908.

54. Um JH, et al. Activation of 5'-AMP-activated kinase with diabetes drug metformin induces casein kinase lepsilon (CKlepsilon)-dependent degradation of clock protein mPer2. J Biol Chem. 2007:282:20794-8.

55. Zhu $Y$, et al. Non-synonymous polymorphisms in the circadian gene NPAS2 and breast cancer risk. Breast Cancer Res Treat. 2008;107:421-5.

56. Rossetti S, Corlazzoli F, Gregorski A, Azmi NHA, Sacchi N. Identification of an estrogen-regulated circadian mechanism necessary for breast acinar morphogenesis. Cell Cycle Georget Tex. 2012;11:3691-700.

57. Akhtar N, Streuli CH. An integrin-ILK-microtubule network orients cell polarity and lumen formation in glandular epithelium. Nat Cell Biol. 2013;15:17-27.

58. Gery S, Virk RK, Chumakov K, Yu A, Koeffler HP. The clock gene Per2 links the circadian system to the estrogen receptor. Oncogene. 2007;26:7916-20.

59. Green KA, Carroll JS. Oestrogen-receptor-mediated transcription and the influence of co-factors and chromatin state. Nat Rev Cancer. 2007:7:713-22.

60. Teboul M, Gréchez-Cassiau A, Guillaumond F, Delaunay F. How nuclear receptors tell time. J Appl Physiol Bethesda Md. 2009;1985(107):1965-71.

61. Jiang $W$, et al. The circadian clock gene Bmal1 acts as a potential antioncogene in pancreatic cancer by activating the p53 tumor suppressor pathway. Cancer Lett. 2016;371:314-25.

62. Climent J, et al. Deletion of the PER3 gene on chromosome 1p36 in recurrent ER-positive breast cancer. J Clin Oncol Off J Am Soc Clin Oncol. 2010;28:3770-8

63. Davis S, Mirick DK, Stevens RG. Night shift work, light at night, and risk of breast cancer. J Natl Cancer Inst. 2001;93:1557-62.

64. Hansen J. Increased breast cancer risk among women who work predominantly at night. Epidemiol Camb Mass. 2001;12:74-7.

65. Megdal SP, Kroenke CH, Laden F, Pukkala E, Schernhammer ES. Night work and breast cancer risk: a systematic review and meta-analysis. Eur J Cancer Oxf Engl. 2005;1990(41):2023-32.

66. Schernhammer ES, Kroenke $\mathrm{CH}$, Laden F, Hankinson SE. Night work and risk of breast cancer. Epidemiol Camb Mass. 2006;17:108-11.

67. Stevens RG. Light-at-night, circadian disruption and breast cancer: assessment of existing evidence. Int J Epidemiol. 2009:38:963-70.

68. Haus EL, Smolensky MH. Shift work and cancer risk: potential mechanistic roles of circadian disruption, light at night, and sleep deprivation. Sleep Med Rev. 2013;17:273-84. 
69. Richter K, et al. Recommendations for the prevention of breast cancer in shift workers. EPMA J. 2011;2:351-6.

70. Menegaux F, et al. Night work and breast cancer: a population-based casecontrol study in France (the CECILE study). Int J Cancer. 2013;132:924-31.

71. Hurley S, et al. Light at night and breast cancer risk among California teachers. Epidemiol Camb Mass. 2014;25:697-706.

72. Van Dycke KCG, et al. Chronically Alternating Light Cycles Increase Breast Cancer Risk in Mice. Curr Biol CB. 2015;25:1932-7.

73. Schernhammer ES, et al. Rotating night shifts and risk of breast cancer in women participating in the nurses' health study. J Natl Cancer Inst. 2001;93: 1563-8.

74. Brainard GC, Richardson BA, King TS, Reiter RJ. The influence of different light spectra on the suppression of pineal melatonin content in the Syrian hamster. Brain Res. 1984;294:333-9.

75. Blask DE, Dauchy RT, Sauer LA, Krause JA, Brainard GC. Growth and fatty acid metabolism of human breast cancer (MCF-7) xenografts in nude rats: impact of constant light-induced nocturnal melatonin suppression. Breast Cancer Res Treat. 2003;79:313-20.

76. Hill SM, et al. Melatonin: an inhibitor of breast cancer. Endocr Relat Cancer. 2015;22:R183-204

77. Dauchy RT, et al. Circadian and melatonin disruption by exposure to light at night drives intrinsic resistance to tamoxifen therapy in breast cancer. Cancer Res. 2014;74:4099-110.

78. Bracci $M$, et al. Rotating-shift nurses after a day off: peripheral clock gene expression, urinary melatonin, and serum 17- $\beta$-estradiol levels. Scand J Work Environ Health. 2014:40:295-304.

79. Kochan DZ, et al. Circadian disruption-induced microRNAome deregulation in rat mammary gland tissues. Oncoscience. 2015;2:428-42.

80. Kochan DZ, et al. Circadian-disruption-induced gene expression changes in rodent mammary tissues. Oncoscience. 2016;3:58-70.

81. Gautherie M, Gros C. Circadian rhythm alteration of skin temperature in breast cancer. Chronobiologia. 1977;4:1-17.

82. Mormont MC, Lévi F. Circadian-system alterations during cancer processes: a review. Int J Cancer J Int Cancer. 1997;70:241-7.

83. Schubauer-Berigan MK, et al. Breast cancer incidence in a cohort of U.S. flight attendants. Am J Ind Med. 2015;58:252-66.

84. Stevens RG, Brainard GC, Blask DE, Lockley SW, Motta ME. Breast cancer and circadian disruption from electric lighting in the modern world. CA Cancer J Clin. 2014:64:207-18. 